Address for Correspondence: Xiao-ning Zhong,

Department of Respiratory Medicine, First Affiliated Hospital of Guangxi Medical University, Nanning 530021, Guangxi Zhuang Autonomous Region, China E-mail: xnzhong101@sina.com

\begin{tabular}{|l|}
\hline Access this article online \\
\hline Website: \\
www.intern-med.com \\
\hline DOI: \\
10.1515/jtim-2015-0015 \\
\hline Quick Response Code: \\
\hline \\
\\
\\
\end{tabular}

\title{
Current status and inspiration on macrolides in the treatment of chronic obstructive pulmonary disease
}

\author{
Shi-lin Qiu, Xiao-ning Zhong \\ Department of Respiratory Medicine, First Affiliated Hospital of Guangxi Medical University, Nanning 530021, \\ Guangxi Zhuang Autonomous Region, China
}

\section{INTRODUCTION}

Chronic obstructive pulmonary disease (COPD) is associated with an enhanced chronic inflammatory response to noxious particles or gases in the airways and the lungs. Immunoreactions participate in the reinforcement and increase of COPD inflammation. The Global Initiative for Chronic Obstructive Lung Disease (GOLD) in 2014 proposed that the target of drug therapy is to relieve symptoms, reduce acute exacerbations, and improve quality of life and exercise endurance. In the stable phase, drug therapy for COPD patients at all levels centers on bronchial dilation agents, such as LABA (long-acting beta2agonist) and LAMA (long-acting muscarinic antagonist). Glucocorticoids used alone are not recommended in current solution but can be used in combination only for patients classified as $\mathrm{C}$ and $\mathrm{D}$ (patients who have high risk of acute exacerbations). The above suggests the limitation and lagging of current anti-inflammatory treatment. Until now, there has not been a drug that can defer the chronic decline of pulmonary function or that can lower fatality rate and reduce incidence of complications. For this reason, early and effective antiinflammatory management are concerned issues. Compared with bronchial asthma patients, due to resistance to hormones, COPD patients have unsatisfying antiinflammatory responses to conventional glucocorticoids. Now that present antiinflammatory drugs are not enough to satisfy clinical demands, novel antibiotics and therapeutic targets are greatly expected.

Macrolides, containing 14-membered ring, 15-membered ring, and 16-membered ring, are a kind of antibiotics separated from Streptomyces. Their main function is to suppress bacterial protein synthesis. The 14- and 15-membered rings are not only antibiotic but also anti-inflammatory and immunomodulatory, suppressing the neutrophils and macrophages in particular. Ever since the 1980s, the successful application of erythromycin in the treatment of diffuse pan bronchiolitis (DPB) has made people pay attention to the antiinflammatory function of macrolides. As a result, they have been applied in the management of other airway chronic inflammation diseases such as COPD, bronchiectasis, and bronchial asthma. Thus, we will present a discussion on current research status and inspiration on macrolides in the treatment of COPD.

\section{BASIC RESEARCH ON MACROLIDES' ANTI- INFLAMMATORY AND IMMUNOMODULATORY FUNCTION}

Previous studies have shown that through reducing the amount of pro-inflammatory factors, chemokines and all kinds of adhesive factors, macrolides can lessen the aggregation of neutrophils and macrophages in airways so that they eventually produce an anti-inflammatory effect. ${ }^{[1]}$ Latest animal experiments show that in the pulmonary emphysema model, intervention with erythromycin can relieve inflammation and emphysema in airways, lung parenchyma, and pulmonary vessels caused by exposure to cigarette smoke. Further research shows 
that in erythromycin-treated mice that were exposed also to cigarette smoke, the numbers of neutrophils, lymphocytes, and macrophages were significantly lower in the cellular content of bronchoalveolar lavage fluid than those in mice exposed only to cigarette smoke. Moreover, various inflammation mediators and enzymes were also decreased significantly, such as matrix metalloproteinase (MMP)-2, MMP-9, TNF-alpha, and IL-6. ${ }^{[2-4]}$ In vitro study shows that the anti-inflammatory effect of erythromycin is developed by suppressing the activity of $\mathrm{H}_{2} \mathrm{O}_{2}$-induced human bronchial epithelial cells transcription factors, such as activator protein 1 (AP-1) and nuclear factor (NF)-kappa B. ${ }^{[5]}$ Besides, erythromycin has preferable inhibiting effect on inflammation and rebuilding of the pulmonary vessels in emphysema mice. ${ }^{[6]}$ Our recent research found imbalance of lymphocytes such as Th1/Tc1, Th17/Tc17, and Treg in cigarette smoke-induced emphysema mice, while the balance could not be restored after separation from the exposure. ${ }^{[7]}$ In cigarette smoke-induced emphysema mice, after treatment with erythromycin, the Treg imbalance was corrected to some degree in lung parenchyma, indicative of erythromycin's immunomodulatory effect. ${ }^{[4]}$

Hormonal resistance is present in COPD patients, as compared with bronchial asthma patients. Moreover, large doses of glucocorticoids cannot suppress their lung inflammation. The reason for this hormonal resistance may be that in the smoking condition, oxidative stress and nitrate activate NF-kappa B and P13K, resulting in lowered expression and decreased activity of histone deacetylase $2 .{ }^{[8]}$ Recent in vitro study shows that erythromycin downregulated the expression of cigarette extractivestimulated human macrophages NF-kappa B, leading to upregulated expression and activity of histone deacetylase $2{ }^{\left[{ }^{[9]}\right.}$ The above fundamental research shows that macrolides are anti-inflammatory as well as immunomodulatory.

\section{CLINICAL RESEARCH ON MACROLIDES IN COPD}

The pathological feature of COPD is chronic inflammation in airways and lungs, characterized by infiltration of neutrophils and macrophages. Macrolides can significantly reduce the release of neutrophil inflammatory mediators, thus theoretically being helpful in the control of chronic inflammation in COPD. In recent years, several randomized controlled trials (RCTs) show that macrolides have a definite effect on acute exacerbations of COPD. Seemungal et al. ${ }^{[10]}$ applied erythromycin in the treatment of 109 COPD patients in 12 months, and they found that the exacerbation frequency of erythromycin group was reduced significantly than that of the contrast group, but it had no effect on forced expiratory volume in the first second $\left(\mathrm{FEV}_{1}\right)$ and inflammatory factors in blood and phlegm, such as IL-6, IL-8, and C-reactive protein (CRP). In our research with $31 \mathrm{COPD}$ patients, we observed that after the 6-month treatment of erythromycin (125 mg per time, 3 times per day) COPD exacerbation frequency was reduced and the level of neutrophil elastases was lowered in the phlegm of COPD patients, as well as the number of neutrophils was decreased in phlegm. ${ }^{[11]} \mathrm{A}$ larger RCT on COPD was performed; 1142 were randomly assigned to receive azithromycin, at a dose of $250 \mathrm{mg}$ daily (570 participants), or placebo (572 participants) for 1 year. And the hazard ratio for having an acute exacerbation of COPD per patient-year in the azithromycin group was 0.73 . The frequency of exacerbations was 1.48 exacerbations per patient-year in the azithromycin group, as compared with 1.83 per patient-year in the placebo group. And the median time to the first exacerbation was 266 days among participants receiving azithromycin, as compared with 174 days among participants receiving placebo. ${ }^{[12]}$ Azithromycin improved quality of life. Patients with a diagnosis of COPD who had received treatment for three or more exacerbations in the previous year were randomly assigned to receive $500 \mathrm{mg}$ azithromycin or placebo three times a week for 12 months. Azithromycin resulted in a significant reduction in the exacerbation rate versus placebo. The exacerbation rate per patient per year was 1.94 for the azithromycin group and 3.22 for the placebo group. Azithromycin also significantly postponed the first-time acute exacerbation. The first exacerbation day was 130 for the azithromycin group and 59 for the placebo group. ${ }^{[13]}$ In a latest metaanalysis, macrolides were safe and effective in reducing the acute exacerbation of COPD and benefit more significantly when continuously applied to patients for 6 months. ${ }^{[14]}$

The above research studies show that macrolides reduce inflammatory index such as neutrophil elastase in COPD patients' airways, lower acute exacerbation frequency, and improve life quality for COPD patients.

\section{CONCERNED PROBLEMS AND APPLICATION PROSPECT OF MACROLIDES IN COPD}

The anti-inflammatory function of macrolides has been recognized, but still the target population who may benefit most and the proper timing in COPD remain unclear. Uzun et al. ${ }^{[13]}$ found that azithromycin maintenance treatment was most beneficial for patients with a diagnosis of COPD who had had three or more exacerbations in the previous year. Besides, as for the moderate COPD patients who were older or using antibiotics or steroids, the use of azithromycin can effectively reduce the acute severe exacerbations. ${ }^{[15]}$ Simpson et al. ${ }^{[16]}$ reported that in stable COPD with neutrophilic bronchitis, the use of azithromycin $250 \mathrm{mg}$ daily for 12 weeks reduced severe 
exacerbations, sputum neutrophils, CXCL8 levels, and bacterial load. According to the 2014 GOLD guideline, a new content of COPD with comorbid bronchiectasis was added to the chapter of complications. With the application of CT scanning in COPD evaluation, bronchiectasis that already existed yet cannot be detected now can be identified. These imaging changes are associated with longer course of disease and increased mortality rate of COPD. When treating bronchiectasis of COPD patients, commonly used COPD medicines are recommended to be added on the basis of bronchiectasis. Altenburg et al. ${ }^{[17]}$ discovered that the use of azithromycin ( $250 \mathrm{mg}$ per day) for 12 months significantly decreased the risk of acute exacerbations in bronchiectasis. Similarly, Serisier et al. ${ }^{[18]}$ observed that the use of erythromycin (400 $\mathrm{mg}$ per day) for 2 months also significantly reduced the risk of acute exacerbations in bronchiectasis. Thus, in treating COPD with comorbid bronchiectasis, the use of macrolides may be more beneficial. In subgroup analyses, it is shown that macrolides behave better in treating COPD patient whose lung function is GOLD II or III, ${ }^{[12,13,15]}$ suggesting that macrolides may have a better anti-inflammatory effect in the early stage of COPD. Even so, which subgroup of COPD suits the most for long-term application and when to use macrolide therapy still require further observation.

The therapeutic effect of macrolides on COPD has been verified, but side effects and safety problems of long-term administration are getting increasing attention. Commonly seen side effects of macrolides include gastrointestinal reaction, hepatic impairment, and hearing impairment. Major cardiac toxicity includes torsades de pointes and QT prolongation. It is more likely to happen in patients who already suffer from QT prolongation, hypokalemia, hypocalcemia, have below average heart rate or are using anti-arrhythmia medicines. Ray et al. ${ }^{[19]}$ retrospectively analyzed COPD patients who used azithromycin from 1992 to 2006, and during 5-day azithromycin therapy, as compared with other antibiotic therapy, cardiovascularinduced mortality rate increased. However, Svanstrom et al. ${ }^{[20]}$ reported that among patients aged from 16 to 64, the use of penicillin and azithromycin did not result in increased cardiovascular mortality rate. Mortensen et al. ${ }^{[21]}$ carried out a retrospective cohort study, which compared patients who were aged over 65 years and hospitalized with pneumonia from 2002 through 2012 and were prescribed azithromycin therapy and patients receiving other antibiotic therapy. Their findings showed that as compared with other antibiotic treatment, treatment with azithromycin was associated with a lower risk of 90-day mortality. However, they found significantly increased odds of myocardial infarction but not any cardiac arrhythmias or heart failure. Current documents show that patients who suffer from cardiac toxicity when using macrolides are those who already have other cardiac risk factors. Therefore, it is suggested that during the therapy, cardiac risk factors and basic condition of the patient should be fully evaluated. Meanwhile electrocardiogram should be performed both before and after the treatment to observe QT time.

Due to the widespread and long-term use of macrolides, possible drug resistance problem is also drawing increasing attention. For COPD patients as compared with the contrast group, the use of azithromycin (250 mg per day) for 12 months increased bacteria resistance to macrolides (0.81 to 0.41). ${ }^{[12]}$ Because of this, the increase of macrolideresistant bacteria (Streptococcus pneumoniae, Haemophilus species, and Moraxella species) among people should get more attention. ${ }^{[2]}$ Thus, to overcome the defect of drug resistance of long-term traditional macrolide therapy, research on new types of anti-inflammatory non-antibiotic macrolides has been taken into account, such as EM703 and CSY0073. ${ }^{[23-24]}$ Currently, the latest research has found a new type of macrolide medicine called solithromycin (CEM-101). Compared with other traditional macrolide medicines, CEM-101 is highly anti-inflammatory and recovers the patients' sensitivity to steroids by inhibiting NF-kappa B and P13K. ${ }^{[25-26]}$

The synergistic effect of combination therapy with macrolides and other anti-inflammatory drugs also deserves attention. Previous research studies suggest that macrolides might recover COPD patients' sensitivity to steroids. Our in vitro study finds that the combination of erythromycin and dexamethasone reduces the amount of IL-8 produced by peripheral blood mononuclear cells and U937 cells stimulated by cigarette extractive, thus producing its anti-inflammatory effect (unpublished). Besides, we have also discovered that by reducing the expression of costimulatory molecules CD40 on the surface of dendritic cells, erythromycin inhibits cigarette smoke extractive from stimulating dendritic cells, inducing $\mathrm{CD}^{+}{ }^{+} \mathrm{T}$ cells differentiation into Th17 cells (unpublished). Considering that dendritic cells are the major antigen-presenting cells, which connect innate immunity and acquired immunity, this might be a new target spot for future COPD antiinflammatory treatment.

\section{CONCLUSION}

Although the function of macrolides in the treatment of COPD has already turned from fundamental research and small-sampled clinical research into large-scale multicentered clinical research, and gladly these research all suggest that macrolides have unique anti-inflammatory function in COPD, the mechanism still remains unclear, and suitable COPD subgroups, staging and course of treatment still need further observation. Compared with currently 
widespread LAMA, LABA, and inhaled glucocorticoids, the status of macrolide therapy in COPD has not yet reached consensus. Further fundamental research and more largescale multi-centered clinical research are needed.

\section{Conflicts of Interest}

None declared.

\section{REFERENCES}

1. Kanoh S, Rubin BK. Mechanisms of action and clinical application of macrolides as immunomodulatory medications. Clin Microbiol Rev 2010; 23: 590-15.

2. Mikura S, Wada H, Higaki M, Yasutake T, Ishii H, Kamiya S, et al. Erythromycin prevents the pulmonary inflammation induced by exposure to cigarette smoke. Transl Res 2011; 158: 30-7.

3. Zhou Y, Tan X, Kuang W, Liu L, Wan L. Erythromycin ameliorates cigarette smoke-induced emphysema and inflammation in rats. Transl Res 2012 ; 159: 464-72.

4. Bai J, Qiu SL, Zhong XN, Huang QP, He ZY, Zhang JQ, et al. Erythromycin enhances CD4+Foxp3+ regulatory T-cell responses in a rat model of smoke-induced lung inflammation. Mediators Inflamm 2012; 2012: 410232.

5. He Z, Li B, Yu L, Liu Q, Zhong N, Ran P. Suppression of oxidantinduced glutathione synthesis by erythromycin in human bronchial epithelial cells. Respiration 2008; 75: 202-9.

6. Zhong XN, Guo SM. An experimental study on pulmonary vascular inflammation in a rat model of chronic bronchitis and emphysema. Chin J Tuberc Respir Dis 2006; 29: 435-9.

7. Duan MC, Tang HJ, Zhong XN, Huang Y. Persistence of Th17/Tc17 cell expression upon smoking cessation in mice with cigarette smokeinduced emphysema. Clin Dev Immunol 2013; 2013: 350727.

8. Yin C, Wang LM, Meng XY. Clinical observation of low dose theophylline combined with inhaled corticosteroids in the treatment of chronic obstructive pulmonary disease. Med J Chin PLA 2007; 32: 777.

9. Li M, Zhong X, He Z, Wen M, Li J, Peng X, et al. Effect of erythromycin on cigarette-induced histone deacetylase protein expression and nuclear factor-kappaB activity in human macrophages in vitro. Int Immunopharmacol 2012; 12: 643-50.

10. Seemungal TA, Wilkinson TM, Hurst JR, Perera WR, Sapsford RJ, Wedzicha JA. Long-term erythromycin therapy is associated with decreased chronic obstructive pulmonary disease exacerbations. Am J Respir Crit Care Med 2008; 178: 1139-47.

11. He ZY, Ou LM, Zhang JQ, Bai J, Liu GN, Li MH, et al. Effect of 6 months of erythromycin treatment on inflammatory cells in induced sputum and exacerbations in chronic obstructive pulmonary disease. Respiration 2010; 80: 445-52.

12. Albert RK, Connett J, Bailey WC, Casaburi R, Cooper JAD, Criner GJ, et al. Azithromycin for prevention of exacerbations of COPD. N Engl J Med 2011; 365: 689-98.
13. Uzun S, Djamin RS, Kluytmans JA, Mulder PG, van't Veer NE, Ermens AA, et al. Azithromycin maintenance treatment in patients with frequent exacerbations of chronic obstructive pulmonary disease(COLUMBUS): a randomised, doubleblind, placebo-controlled trial. Lancet Respir Med 2014; 2: 361-8.

14. Yao GY, Ma YL, Zhang MQ, Gao ZC. Macrolide therapy decreases chronic obstructive pulmonary disease exacerbation: a meta-analysis. Respiration 2013; 86: 254-60.

15. Han MK, Tayob N, Murray S, Dransfield MT, Washko G, Scanlon PD, et al. Predictors of chronic obstructive pulmonary disease exacerbation reduction in response to daily azithromycin therapy. Am J Respir Crit Care Med 2014; 189: 1503-8.

16. Simpson JL, Powell H, Baines KJ, Milne D, Coxson HO, Hansbro P. The effect of azithromycin in adults with stable neutrophilic COPD: a double blind randomised, placebo controlled trial. PLoS One 2014; 9: e105609.

17. Altenburg J, de Graaff CS, Stienstra Y, Sloos JH, van Haren EH, Koppers RJ, et al. Effect of azithromycin maintenance treatment on infectious exacerbations among patients with non-cystic fibrosis bronchiectasis: the BAT randomized controlled trial. JAMA 2013;309: 1251-9.

18. Serisier DJ, Martin ML, McGuckin MA, Lourie R,Chen AC,Brain $B$, et al. Effect of long-term, low-dose erythromycin on pulmonary exacerbations among patients with non-cystic fibrosis bronchiectasis: the BLESS randomized controlled trial. JAMA 2013; 309: 1260-7.

19. Ray WA, Murray KT, Hall K, Arbogast PG, Stein CM. Azithromycin and the risk of cardiovascular death. N Engl J Med 2012;366: 1881-90.

20. Svanström HH, Pasternak B, Hviid A. Use of azithromycin and death from cardiovascular causes. N Engl J Med 2013; 368: 1704-12.

21. Mortensen EM, Halm EA, Pugh MJ, Copeland LA, Metersky M, Fine MJ, et al. Association of azithromycin with mortality and cardiovascular events among older patients hospitalized with pneumonia. JAMA 2014; 311: 2199-208.

22. Serisier DJ. Risks of population antimicrobial resistance associated with chronic macrolide use for inflammatory airway diseases. Lancet Respir Med 2013; 1: 262-74.

23. Li YJ, Shimizu T, Hirata $Y$, Inagaki $H$, Takizawa $H$, Azuma A, et al. EM, EM703 inhibit NF-kB activation-induced by oxidative stress from diesel exhaust particle in human bronchial epithelial cells: importance in IL-8 transcription. Pulm Pharmacol Ther 2013; 26:318-24.

24. Mencarelli A, Distrutti E, Renga B, Cipriani S, Palladino G, Booth $C$, et al. Development of nonantibiotic macrolide that corrects inflammation-driven immune dysfunction in models of inflammatory bowel diseases and arthritis. Eur J Pharmacol 2011; 665:29-39.

25. Kobayashi Y, Wada H, Rossios C, Takagi D, Charron C, Barnes PJ, et al. A novel macrolide/ fluoroketolide, solithromycin (CEM-101), reverses corticosteroid insensitivity via phosphoinositide 3-kinase pathway inhibition. Br J Pharmacol 2013; 169: 1024-34.

26. Kobayashi Y, Wada H, Rossios C, Takagi D, Higaki M, Mikura S, et al . A novel macrolide solithromycin exerts superior anti-inflammatory effect via NF-kappaB inhibition. J Pharmacol Exp Ther 2013;345:76-84.

How to cite this article: Qiu S, et al. Current status and inspiration on macrolides in the treatment of chronic obstructive pulmonary disease. J TransI Intern Med 2015;3:85-88 